\title{
ARSM: Auto Rate Selection Multicast Mechanism for Multi-rate Wireless LANs*
}

\author{
José Villalón, Yongho Seok, Thierry Turletti, Pedro Cuenca, \\ and Luis Orozco-Barbosa \\ Instituto de Investigación en Informática de Albacete, \\ Universidad de Castilla-La Mancha, 02071 Albacete, Spain \\ \{josemvillalon, pcuenca, lorozco\}@dsi.uclm.es \\ Institut National de Recherche en Informatique et en Automatique, INRIA \\ Sophia Antipolis, France \\ \{Yongho.Seok, Thierry.Turletti\}@sophia.inria.fr
}

\begin{abstract}
Multicast is an efficient paradigm for transmitting data from a sender to a group of receivers. The IEEE 802.11 wireless LANs standards specify how to send multicast frames with no ACK and using one of the Basic Service Set (BSS) rates. This situation has led many researchers to design techniques aiming to improve reliability of a multicasting mechanism. The Leader-Base Protocol (LBP) is one such mechanism proposed in the literature that is the most promising approach. The main idea behind the design of the LBP mechanism is to reduce the probability of collision of the feedback messages sent by the multicast group members. However, the LBP mechanism falls short by not considering the varying conditions characterizing the wireless channels. In this paper, we introduce a novel auto rate selection multicast mechanism for multi-rate wireless LANs, namely ARSM (Auto Rate Selection for Multicast), capable of adapting the data transmission to the varying conditions of the channel. Our simulation results show that our new scheme outperforms the IEEE 802.11 and the LBP mechanisms.
\end{abstract}

\section{Introduction}

The IEEE 802.11 Media Access Control (MAC) protocol provides a physical-layer multi-rate capability [1]. The original IEEE 802.11 protocol supports a single base rate, typically $2 \mathrm{Mbps}$. With the multi-rate enhancement, the data transmission can take place at various rates according to the channel conditions. Higher data rates than the base rate are possible when the Signal-to-Noise Ratio (SNR) is sufficiently high. Within the IEEE 802.11a standard [2] the set of possible data rates are 6, 9, 12, 18, 24, 36, 48 and $54 \mathrm{Mbps}$ whereas for the IEEE 802.11b standard [3] the set of possible data rates includes 1, 2, 5.5 and $11 \mathrm{Mbps}$. Since the multi-rate enhancements are

This work was supported by the Ministry of Science and Education of Spain under CICYT project TIC2003-08154-C06-02, the Council of Science and Education of Castilla-La Mancha under project PAI06-0106, FEDER and the Korea Research Foundation Grant funded by the Korean Government (MOEHRD) (KRF- 2005-214-D00340). 
implemented into the physical layer, the MAC mechanisms should be adapted in order to fully exploit them. The Auto Rate Fallback (ARF) protocol is the most known commercial implementation of the IEEE 802.11 MAC making use of this feature [4]. Under the ARF protocol, after the reception of ten consecutive Acknowledgements (ACK), the next higher mode is selected for future data frames. If the delivery of the eleventh frame is unsuccessful, it immediately falls back to the previously supported mode. During other cycles with less than ten consecutive ACKs, it switches to a lower rate mode after two successive ACK failures.

Since the ARF protocol selects the data rate taking into account the channel conditions between the Access Point (AP) and a given Mobile Terminal (MT), it is only suitable for point-to-point communications. In the case of point-to-multipoint communications, i.e., multicast and broadcast services, it is more difficult to determine the highest data rate to be used since the channel conditions between the AP and each one of the MTs in the multicast group may differ and no feedback is available. In most current setups, it comes to the network administrator to setup the data rate to be used by the point-to-multipoint service. This rate is then used to provide network connectivity to all the MTs covered by the AP. It is obvious that in order to ensure full coverage, the rate to be used is determined by using the channel conditions between the AP and the MT exhibiting the worst channel conditions. Furthermore, since the coverage of the AP is inversely proportional to the transmission data rate, the administrator should then select the proper data rate according to the distance between the AP and the worst MT. As the distance increases, the data rate has to be reduced in order to compensate for the increased range that the AP has to cover. This simple approach does not efficiently support the point-to-multipoint communications service.

In this paper, we introduce a novel auto rate selection multicast mechanism for multi-rate wireless LAN, from now on referred as the ARSM mechanism, capable of adapting the data transmission to the varying conditions of the channel. The remainder of this paper is organized as follows. We start Section 2 by providing some background on the issues to be addressed on the design of multicast services to be deployed in a multi-rate wireless LAN. The proposed ARSM mechanism is described in Section 3. Section 4 presents simulation results. Section 5 concludes the paper.

\section{Background}

In IEEE 802.11 wireless LANs, multicasting is specified as a simple broadcasting mechanism that does not make use of ACK frames. According to the IEEE 802.11a and IEEE 802.11b standards, all frames with multicast and broadcast Receiver Address (RA) should be transmitted at one of the rates included in the basic rate set.

Most research efforts on multicasting in IEEE 802.11 wireless LANs have focused on improving transmission reliability by integrating ARQ mechanisms into the protocol architecture. In [5], the Leader-Based Protocol (LBP) ARQ mechanism has been proposed to provide the multicast service with some level of reliability. The LBP addresses this issue by assigning the role of group leader to one of the members of the multicast group. The AP designates the MT exhibiting the worst signal quality as group leader. The group leader holds the responsibility to acknowledge the multicast 
packets on behalf of all the multicast group members. Any group member other than the leader MT may issue a Negative Acknowledgement (NACK) only if it detects an error in the transmission process of the multicast packets addressed to the group. The transmission of the NACK may result in a collision with the ACK issued by the group leader. Upon this event, the sender will once again reissue the multicast frame.

In [6], Gupta et al. present a reliable multicast MAC protocol, namely the 802.11MX protocol. The 802.11 MX uses an ARQ mechanism supplemented by a busy tone signal. When an MT associated to a multicast group receives a corrupted packet, it sends a NACK tone instead of actually transmitting a NACK frame. Upon detecting the NACK tone, the sender will retransmit the data packet. On the contrary, if the AP does not detect the NACK tone, the AP assumes that the transmission of the multicast packet has been successfully completed. Since the $802.11 \mathrm{MX}$ mechanism does not need a leader to operate, it performs better than the LBP protocol in terms of both data throughput and reliability. However, this mechanism falls short on addressing events when some of the group members do not properly receive a packet. For instance, in the event that the header of a multicast packet may get corrupted, a group member will be unable to detect it and signal this event.

It should be clear that the mechanisms above described only focus on solving the reliability of the multicast service in wireless LANs. They do not adapt the transmission rate of the multicast packet taking into account the quality of the signal received by each and every member of the multicast group. To the best of our knowledge, there is no related work to provide an auto rate adaptation mechanism for the multicast service over wireless LANs. In this paper, we make use of the multirate capabilities present in the physical layer of the latest IEEE 802.11 wireless LANs for developing a reliable multicast service.

\section{Auto Rate Selection for Multicast (ARSM)}

The ultimate goal of the ARSM protocol to be introduced herein is to enable the deployment of a reliable and efficient multicast protocol to be integrated into the protocol architecture of multirate wireless networks. By efficient, we mean that the overhead required by the ARSM to operate should be kept to minimum levels. ARSM enables the exchange of information pertaining to the physical channel conditions as perceived by each and every MT. This information can be used by ARSM to determine the transmission rate accordingly. In the following sections, we introduce the various mechanisms making part of ARSM.

\subsection{Multicast Channel Probe Operation (MCPO) of ARSM}

ARSM is an adaptive mechanism in which the AP selects the PHY data rate to be used for multicast data transmission. The PHY data rate to be used is determined by taking into account the channel conditions perceived by each and every MT belonging to a given multicast group. Under the proposed scheme, the AP starts by multicasting a control frame, namely the Multicast Probe (MP) frame, to the multicast group members. Upon receiving the MP frame, each multicast member estimates the SNR of the channel, i.e., the quality of the wireless medium. Based on the SNR, each MT 
will determine the point in time for replying to the AP. According to the proposed mechanism, the MT having detected the lowest SNR will be the one in charge of first replying to the AP, by issuing a Multicast Response (MR) frame. Upon detecting the transmission of the reply and in the absence of errors, all the other group members should normally refrain from replying to the AP. The AP then selects the multicast data rate based on the SNR of the reporting MT.

\begin{tabular}{|c|c|c|c|c|c|c|c|}
\hline 2 & 2 & 6 & 6 & 6 & 1 & 4 \\
\hline $\begin{array}{c}\text { Frame } \\
\text { Control }\end{array}$ & Duration & $\begin{array}{c}\text { Dest } \\
\text { Address }\end{array}$ & $\begin{array}{c}\text { Source } \\
\text { Address }\end{array}$ & BSSID & $\begin{array}{c}\text { Sequence } \\
\text { Control }\end{array}$ & SNRleader & FCS \\
\hline
\end{tabular}

(a) Multicast Probe Frame

\begin{tabular}{|c|c|c|c|c|c|c|c|}
\hline 2 & 2 & 6 & 6 & 6 & 2 & 1 \\
\hline $\begin{array}{c}\text { Frame } \\
\text { Control }\end{array}$ & Duration & $\begin{array}{c}\text { Dest } \\
\text { Address }\end{array}$ & $\begin{array}{c}\text { Source } \\
\text { Address }\end{array}$ & BSSID & $\begin{array}{c}\text { Sequence } \\
\text { Control }\end{array}$ & SNRmp & FCS \\
\hline
\end{tabular}

(b) Multicast Response Frame

Fig. 1. Special Multicast Control Frames

Figure 1a shows the format of the MP frame. The duration field of the MP frame is initially set to $C W_{m}$ x SlotTime, where $C W_{m}$ is the length of the contention window, expressed in slots, during which the group members may attempt to transmit the MR frame back to the AP. The destination address field of the MP frame represents the address of the multicast group being addressed by the AP and the $\mathrm{SNR}_{\text {leader }}$ field is set to the SNR received in the latest acknowledgement received by the AP.

After having sent the MP frame, the AP will wait for a period whose length is given by the Short Inter Frame Space (SIFS) parameter of the IEEE 802.11 standard, before changing its interface from transmission mode to listen mode. At the time of sending the multicast frame, the AP starts a timer, namely the MP_timer, initially setting to $\mathrm{CW}_{\mathrm{m}}$ slots. The timer is then decremented by one slot whenever the channel has been sensed idle for a period of time equal to one time slot (SlotTime). On the contrary, whenever the AP detects activity in the channel by means of the Clear Channel Assessment (CCA) mechanism, it immediately freezes the MP_timer.

When a MT receives the MP frame, it checks whether it is a member of this multicast group. If it is not, it sets the NAV parameter to $C W_{m} \mathrm{x}$ SlotTime by using the duration field included in the MP frame. In this way, the MTs that are no members of the multicast group will not interfere with the on-going multicast transmission. Figure $1 \mathrm{~b}$ depicts the format of the MR frame.

In the MR frame, the $\mathrm{SNR}_{\mathrm{mp}}$ field contains the $\mathrm{SNR}$ value of the previously received MP frame. When a MT replies to the AP with an MR frame, an MT uses the backoff mechanism in order to reduce the collision probability with other MR frames. The backoff timer used for transmitting the MR frame is set according to the following expression:

$$
\text { BackoffTimer }=\left\{\begin{array}{rr}
{[0,2]} & S N R_{m p}<S N R_{\text {leader }}-F 1 \\
{[3,5]} & S N R_{\text {leader }}-F 1 \leq S N R_{m p}<S N R_{\text {leader }} \\
{[6,7]} & S N R_{\text {leader }} \leq S N R_{m p}
\end{array}\right.
$$


where $F 1$ is a correcting factor to limit the SNR intervals. From this expression, it is clear that the backoff timer is chosen based on the channel quality of the MT. The MT with the worst SNR chooses the lowest backoff timer and has a chance to transmit the MR frame earlier than all other MTs. In order to reduce the probability of collision of the MR frames, a random number of slots have been assigned to each one of the three intervals. When all the other MTs detect the transmission of the MR frame, all the other MTs refrain from transmitting. In this way, ARSM avoids the MP frame implosion problem.

Following the multicast channel probe operation, the AP selects the appropriate PHY data rate using the feedback information that contains the channel conditions of the MTs. According to the received information, and the value of the MP_timer, the AP could receive three different kinds of feedback information: Explicit Feedback, Implicit Feedback, and No Feedback.

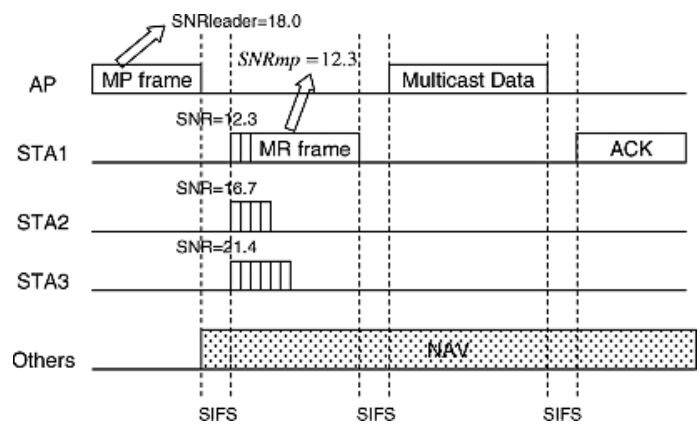

Fig. 2. Explicit Feedback Scenario

Explicit Feedback: the AP receives the MR frame from an MT within the multicast group. In this case, the AP determines the SNR value of the MT with the worst channel quality. Then, it transmits the multicast data frames accordingly. In the scenario depicted in Figure 2, STA1 selects the shortest backoff time since STA1 shows the worst received SNR of MP frame. STA1 then sends the MR frame to the AP after 3 slots; this period is determined through Equation 1.

Implicit Feedback: the AP receives a corrupted MR frame and the MP timer of the AP has not expired. This condition occurs when several MTs reply to the MP frame simultaneously and the MR frames have collided. In this case, the AP can predict an SNR value of MTs with the worst channel quality through the current MP timer value. Through the current MP timer of the AP, the AP identifies the lowest backoff timer among all the MTs in the multicast group. It must be mentioned that the MT with the lowest backoff timer first replies to the AP using an MR frame. The AP should already know the value of the backoff timer chosen by the MT to send MR frame. Using Equation 2, the AP can inversely estimate the SNR range with the lowest backoff timer, where $B T_{m p}$ is the current MP timer value in AP and $\overline{S N R}$ is the estimated worst SNR value. 


$$
\overline{S N R_{m p}}=\left\{\begin{array}{lr}
0 & B T_{m p} \geq 6 \\
S N R_{\text {leader }}-F 1 & 6>B T_{m p} \geq 3 \\
S N R_{\text {leader }} & 3>B T_{m p} \geq 1
\end{array}\right.
$$

Figure 3 shows an example of implicit feedback scenario of the ARSM mechanism. The AP does not receive the MR frame because both MTs, STA1 and STA2, simultaneously have sent an MR to the AP. The MR frames will collide before the MP timer of the AP expires. By using the value of the remaining period of the MP timer of the AP, ARSM is able to estimate the lower bound of MT exhibiting the worst SNR. In this scenario, the worst SNR estimated from the MTs $(\overline{S N R})$ is greater than $15 \mathrm{~dB}$; the AP then chooses the multicast rate corresponding to $15 \mathrm{~dB}$.

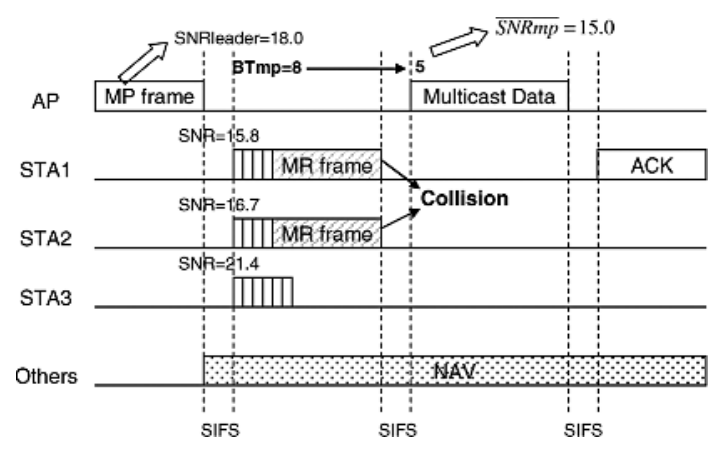

Fig. 3. Implicit Feedback Scenario

No Feedback: The AP does not receive an MR frame and the MP timer of the AP expires. So, none of the MTs in the multicast group reply to the MP frame. This means that either all the MTs in this group have left or that the MP frame has been corrupted during its transmission. In this case, the AP will retransmit the MP frame after waiting for a period of time defined by the DCF backoff mechanism. The number of retransmission attempts for a given MP frame is limited to 4 . When the maximum number of retransmission attempts is selected, the AP assumes that there are no more MTs in the multicast group.

The AP then determines the PHY data rate to be used for the multicast data transmission using the CLARA mechanism [7]. With this mechanism, a MT makes use of the SNR of the feedback signal in order to adapt its data rate to the actual channel conditions. The SNR value is obtained by either explicit feedback $\left(\mathrm{SNR}_{\mathrm{mp}}\right)$ or implicit feedback $(\overline{S N R})$.

The AP can determine the MT exhibiting the worst SNR by using a channel probe mechanism in the absence of a collision involving the MR frame (Explicit Feedback). However, if the MR frame collides (Implicit Feedback), the AP is unable to identify the new leader. In this case, to identify the new leader, the AP will have to send an MP frame before sending the following multicast data frame. The new MP frame to be sent out will set the $\mathrm{SNR}_{\text {leader }}$ field to a negative value. When the MTs in the multicast group receive the MP frame with the $\mathrm{SNR}_{\text {leader }}$ field equal to a negative 
value, only those MTs having sent the previous MR frame (the MTs with smaller SNR) sent to this a new MR frame. Since these MTs will have a very similar SNR, they do not use the backoff mechanism based on the SNR of the received signal, but a random value between $\left[0, \mathrm{CW}_{\mathrm{m}}-1\right]$. This different backoff mechanism is used to further reduce the probability of collision of the MR frames.

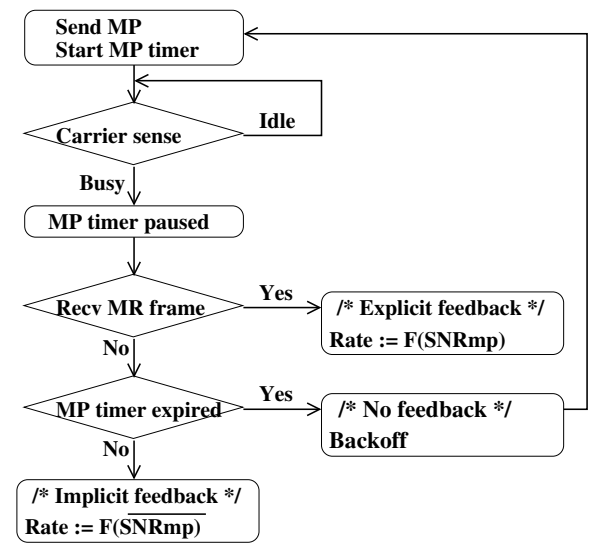

Fig. 4. Multicast Channel Probe Operation (MCPO) of ARSM

Figure 4 shows the MCPO procedure of ARSM. As shown in Figure 4, after having sent an MP frame, the AP will activate its MP timer with the initial value $\mathrm{CW}_{\mathrm{m}}$. The timer will remain active as long as the AP detects that the channel is busy. If the AP receives an error-free MR frame before the timer expires, it will adapt its transmission rate using the explicit feedback. On the contrary, if the AP receives a corrupted MR frame, once its timer expire, the PHY data rate will be selected based on the implicit feedback mechanism.

\subsection{Dynamic Multicast Data Transmission Procedure}

Through the multicast channel probe operation, the AP can estimate the worst SNR of MTs. In order to reduce the amount of processing to be carried out by the MTs, we propose a dynamic multicast data transmission procedure by making use of several multicast data transmissions. Under this scheme, the AP can be found in one of two different states depending on the feedback signals received.

- While the AP successfully delivers multicast data frame, the Multicast Channel Probe Operation is deactivated. In this state, the AP will adapt its PHY data rate using the SNR value contained in the received ACK coming from the group leader using the CLARA mechanism.

- If the AP shows a failure of $N_{t h}$ consecutive multicast transmissions, it initiates the Multicast Channel Probe Operation.

However, this dynamic multicast data transmission needs a mechanism for determining whether the multicast packet has been successfully delivered or not. The IEEE 802.11 standard does not support any mechanism to carry out this verification 
for the multicast service. On this purpose, we use the LBP mechanism. By combining the LBP and CLARA mechanisms, the ARSM mechanism can adapt the data rate taking into account the SNR included in the ACK received from the group leader.

Figure 5 shows an example of the operation of the ARSM mechanism. The mechanism starts by using the MCPO in order to determine the multicast group leader. In this example, the group leader becomes STA1 which is the MT with the lowest SNR value. The AP then turns off the MCPO and starts sending the data frames. After two successful transmissions, it is assumed that STA2 becomes the MT with the worst SNR. This happens at $35 \mathrm{~ms}$ of operation. Since the AP has not become aware of the SNR change of STA2, the AP continues sending the data frames at the same data rate. After the $N_{t h}$ transmission failure, the AP turns on the multicast channel probe operation. With the explicit feedback information from STA3, the AP sets STA3 as the group leader. This happens at $60 \mathrm{~ms}$ of operation.

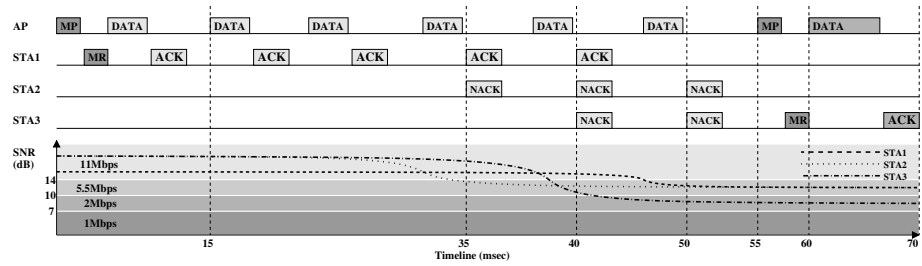

Fig. 5. Dynamic Multicast Data Transmission Procedure

\section{Performance Evaluation}

In this section, we carry out a performance analysis on the effectiveness of our proposed mechanism. Throughout our study, we have made use of the OPNET Modeler tool 11.0 [8], which already integrates the IEEE 802.11 DCF simulator. We have integrated into it the ASRM and the LBP mechanisms.

\subsection{Scenarios and Metrics}

Our performance evaluation has been structured in the following way: first we analyze the performance limitations of the multicast service of the IEEE 802.11 standard. We then evaluate and compare the ARSM and LBP schemes. Towards this end, we have studied the performance of the two schemes by varying the size of the network (coverage area) and using two different multicast group sizes.

In our simulations, we model an IEEE 802.11b WLAN consisting of an AP, several multicast wireless MTs, and five unicast wireless MTs. All MTs are located within a Basic Service Set (BSS), i.e., every MT is able to detect a transmission from any other MT. The access point is located in the center of the BSS, which cell size will be changed throughout the different scenarios under study. The multicast MTs move randomly within the BSS with a constant speed of $5 \mathrm{~km} / \mathrm{h}$, whereas the unicast MTs are static and placed close to the access point. We assume that the unicast packets are always transmitted at 11 Mbps. This setup of the unicast MTs will allow 
us to focus on the evaluation of each one of the multicast schemes under consideration.

For the ARSM scheme, we have set $F l=3$ and $n_{t h}=3$. These values have been determined after an extensive campaign of simulations. By setting $F 1=3$, the number of collisions of the MR frame is considerable reduced. This value corresponds to the distance between the thresholds being used to adapt the transmission rate taking into account the state of the channel. In the case of the value used for $n_{t h}$, we have come to a compromise to limit the number of MP frames to be sent and the time to react to a change on the network. In the case of the $C W$ parameter, we have set it to 8 . This value is fully compliant to the standard and corresponds to the length of the Extended IFS (EIFS) parameter of the IEEE 802.11 standard.

In order to model the wireless channel, we have used the Ricean model to characterize the propagation of the signal throughout the medium [9]. When there is a dominant stationary signal component present, such as a line-of-sight propagation path, the small-scale fading envelope has a Ricean distribution. This is often described in terms of a parameter $k$, which is defined as the ratio between the deterministic signal power and the variance of multi-path fading. If $k$ is equal to 0 , the Ricean distribution reduces to the Rayleigh distribution, in which the signal is only transmitted by reflection. In this work, we have set the parameter $k$ to 32 .

In our scenarios, we have assumed the use of two types of traffic flows: multicast traffic downlink flows and unicast traffic uplink flows. For the downlink traffic, the access point transmits a video stream to the multicast MTs group. For the video streaming source, we have used traces generated from a variable bit-rate H.264 video encoder [10]. We have used the sequence Mobile Calendar encoded on CIF format at a video frame rate of 25 frames/s. The average video transmission rate is around 400 Kbits/s with a packet size equal to 1000 bytes (including RTP/UDP/IP headers). This video application is randomly activated within the interval [1,1.5] seconds from the start of the simulation. In order to limit the delay experienced by the video streaming application, the maximum time that a video packet may remain in the transmission buffer has been set to 2 seconds. Whenever a video packet exceeds these upper bounds, it is dropped. For the unicast traffic, we assume greedy sources. The unicast packet size is equal to 1000 bytes (including the RTP/UDP/IP headers). The unicast sources are also randomly activated within the interval [1,1.5] seconds from the start of the simulation. Throughout our study, we have simulated the two minutes of operation of each particular scenario.

In our simulations, we have started by simulating a WLAN consisting of five unicast MTs and nine multicast MTs. The network size has been initially set to a geographical area of $50 \mathrm{~m} \times 50 \mathrm{~m}$. We have then increased the network size in both dimensions by $10 \mathrm{~m} \times 10 \mathrm{~m}$ to a maximum network size of $140 \mathrm{~m} \times 140 \mathrm{~m}$. Then, the size for the multicast group has been increased to $18 \mathrm{MTs}$.

For the purpose of our performance study, the three metrics of interest are: multicast throughput, unicast throughput, and multicast packet loss rate. The multicast throughput shows the successfully received average data rate by all the multicast MTs. To be able to better evaluate the various schemes with respect to the optimum case, we plot the normalized throughput rather than the absolute throughput. The normalized throughput is calculated with respect to the multicast downlink traffic generated by the AP. The unicast throughput shows the total throughput received by 
the AP from all the unicast MTs. This metric will allow us to estimate the bandwidth not used (available for unicast sources) of each one of the multicast schemes under consideration. Finally the multicast packet loss rate shows the ratio between the packets not having been received by at least a member MT of the multicast group over the total number of packets submitted to the network.

Our measurements started after a warm-up period (about three seconds) allowing us to collect the statistics under steady-state conditions. Each point in our plots is an average over thirty simulation runs, and the error bars indicate $95 \%$ confidence interval.

\subsection{Results}

In the first part of our performance study, we first look at the multicast service as defined by the standard. We have first considered a small-sized network; this setup represents the most manageable of all setups being considered, i.e., the potential number of corrupted packets is limited. Figure 6 shows the results for this first scenario. From the results, it is clear that the standard is unable to effectively provide multicast services. This is due to the fact that the standard does not take any action to recover those packets having been corrupted or lost during their transmission. A loss rate of $18 \%$ is far below all expectations, especially if we do consider the deployment of video streaming applications.

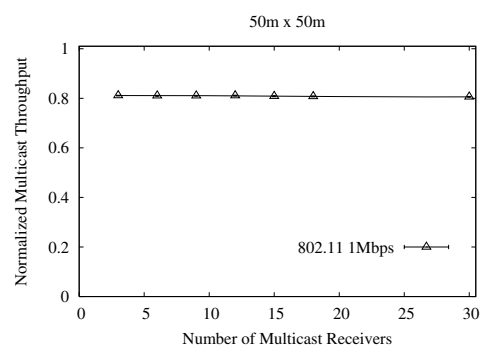

Fig. 6. Limitations IEEE 802.11 Standard for the Multicast Traffic

Figure 7 shows the multicast throughput obtained for both schemes under study. The results depicted in Figure 7 show that ARSM and LBP (1Mbps) schemes are able to provide a reliable multicast for all network sizes. For all the other rates, the performance of LBP decreases as the network size is increased. This is expected since adapting the transmission helps to compensate for the signal impairments due to the distance to be covered by the signal. The figure $7 \mathrm{~b}$ shows the effect to increase the size of multicast group. This figure shows that the performance of LBP mechanisms decreases more quickly when the size of the multicast group is increased.

For the case of the unicast traffic, figure 8 shows that the ARSM outperforms the LBP $1 \mathrm{Mbps}$ for all network sizes. Furthermore, in the case of small-sized network, ARSM is even able to deliver twice the load carried by the LBP $1 \mathrm{Mbps}$ scheme. The figure also shows that the ARSM outperforms the LBP scheme when this latter is able to fully deliver the multicast traffic (see figure 7). The results clearly show the benefits of adapting the transmission rate taking into account the channel conditions. 
The figure $8 \mathrm{~b}$ shows the effect to increase the number of multicast receivers. The results in this figure show that the ARSM scheme is able to cope with large multicast group sizes.

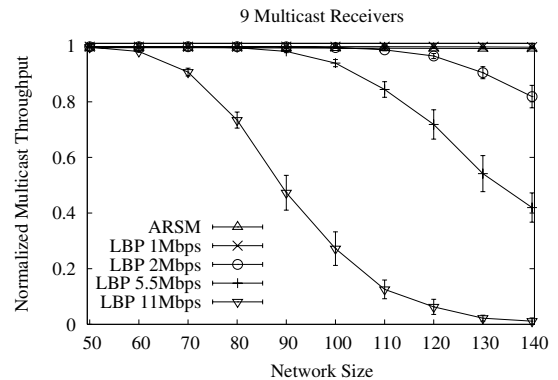

(a)

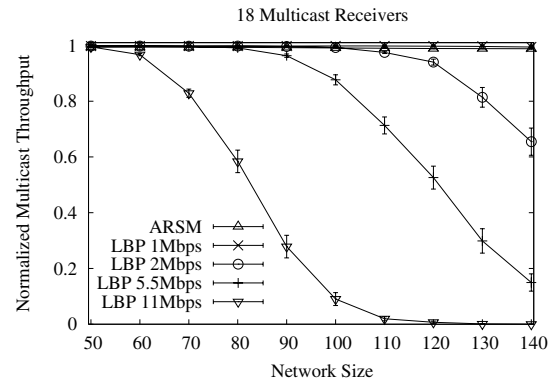

(b)

Fig. 7. Throughput of Multicast Traffic: a) 9 Multicast receivers, b) 18 Multicast receivers

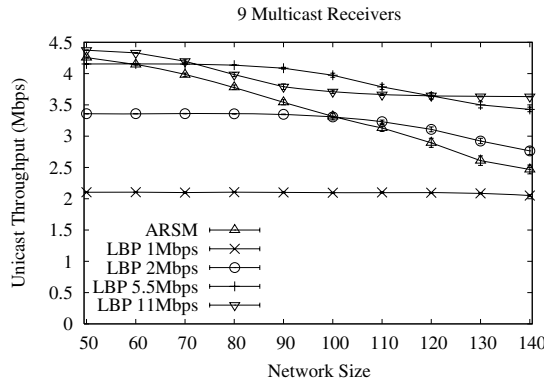

(a)

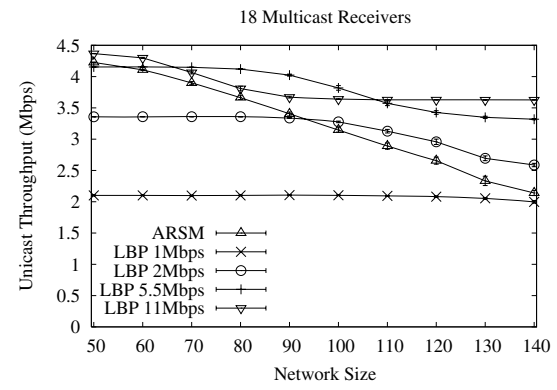

(b)

Fig. 8. Total Throughput of Unicast Traffic: a) 9 Multicast receivers, b) 18 Multicast receivers

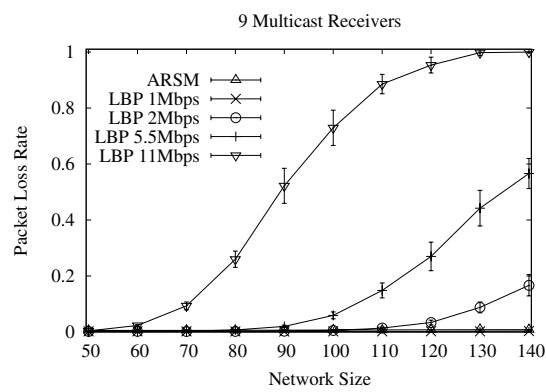

(a)

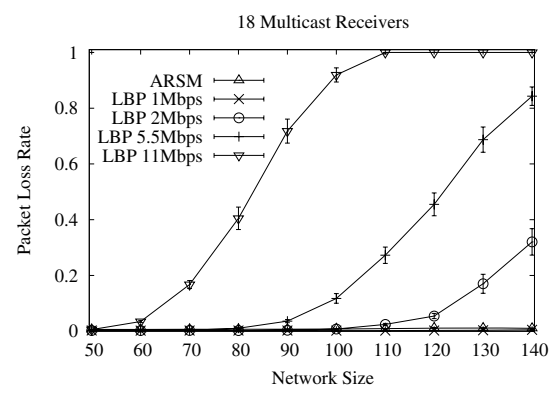

(b)

Fig. 9. Packet Loss Rate of Multicast Traffic: a) 9 Multicast receivers, b) 18 Multicast receivers 
Finally, figure 9 shows the multicast packet loss rate for the ARSM and LBP schemes. This figure shows clearly how the LBP scheme is unable to provide good support to the multicast service for all the network sizes even at rates as low as 2 Mbps. The figure shows that only ARSM and LBP 1Mbps schemes are able to provide a reliable multicast for all network sizes.

\section{Conclusions}

We have proposed an adaptive IEEE 802.11 multicast protocol design that takes into account the dynamic channel conditions. The mechanism requires knowing the operating conditions of the channel as perceived by the multicast group members. The transmission rate to be used for the multicast traffic is determined based on the feedback received by the group leader. We have also paid particular attention to limit the overhead introduced by the multicast rate adaptation mechanism. We have carried out an extensive campaign of simulations aiming to analyze the impact of various key parameters, mainly the network size and the size of the multicast group, over the performance of the proposed scheme. Our results have shown that the ARSM mechanism outperforms the IEEE 802.11 and LBP mechanisms.

\section{References}

1. LAN MAN Standards Committee of the IEEE Computer Society, ANSI/IEEE Std 802.11, "Part 11: Wireless LAN Medium Access Control (MAC) and Physical Layer (PHY) Specifications", 1999 Edition.

2. LAN MAN Standards Committee of the IEEE Computer Society, ANSI/IEEE Std 802.11, "Part 11: Wireless LAN Medium Access Control (MAC) and Physical Layer (PHY) Specifications: High-speed Physical Layer in the 5GHz Band", IEEE 802.11 Standard, 1999.

3. LAN MAN Standards Committee of the IEEE Computer Society, ANSI/IEEE Std 802.11, "Part 11: Wireless LAN Medium Access Control (MAC) and Physical Layer (PHY) Specifications: High-speed Physical Layer Extension in the 2.4 GHz Band", IEEE 802.11 Standard, 1999.

4. A. Kamerman, and L. Monteban, "WaveLAN II: A High-Performance Wireless LAN for the Unlicensed Band", Bell Labs Technical Journal, page 118-133, Summer 1997.

5. Joy Kuri and Sneha Kumar Kasera, "Reliable Multicast in Multi-access Wireless LANs", ACM Wireless Networks, Volume 7, Issue 4, Pages 359 - 369, 2001.

6. S. K. S. Gupta, V. Shankar and S. Lalwani, "Reliable Multicast MAC Protocol for Wireless LANs", IEEE ICC, May 2003.

7. C. Hoffmann, M. H. Manshaei and Thierry Turletti, "CLARA: Closed-Loop Adaptive Rate Allocation for IEEE Wireless LANs", IEEE WIRELESSCOM, June 2005.

8. Opnet.Technologies.Inc. OPNET Modeler 10.0 (c)1987-2004. http://www.opnet.com.

9. Ratish J. Punnoose, Pavel V. Nikitin, and D. Stancil, "Efficient Simulation of Ricean Fading within a Packet Simulator", IEEE Vehicular Technology Conference, 2000.

10. ITU-T Recommendation H.264, "Advanced Video Coding For Generic Audiovisual Services". May 2003. 